\title{
Predictors of Comorbid Psychological Symptoms among Patients with Social Anxiety Disorder after Cognitive-Behavioral Therapy
}

\author{
Sei Ogawa1, Risa Imai1, Masaki Kondo', Toshi A. Furukawa², Tatsuo Akechi' \\ ${ }^{1}$ Department of Psychiatry and Cognitive-Behavioral Medicine, Nagoya City University Graduate School of \\ Medical Sciences, Nagoya, Japan \\ ${ }^{2}$ Department of Health Promotion and Human Behavior, Kyoto University Graduate School of Medicine/School \\ of Public Health, Kyoto, Japan \\ Email: seiogawa1964@nifty.com
}

Received 3 December 2015; accepted 17 January 2016; published 20 January 2016

Copyright (C) 2016 by authors and Scientific Research Publishing Inc. This work is licensed under the Creative Commons Attribution International License (CC BY). http://creativecommons.org/licenses/by/4.0/

(c) (i) Open Access

\begin{abstract}
Aim: The present study aimed to examine the predictors of comorbid psychological symptoms in social anxiety disorder (SAD) after cognitive-behavioral therapy (CBT). Methods: One hundred fourteen SAD patients completed manualized group CBT. We examined associations between the personality dimensions of NEO Five Factor Index (NEO-FFI) and the subscales of Symptom Checklist-90 Revised (SCL-90-R) in SAD patients after CBT using multiple regression analysis. Results: High levels of conscientiousness at baseline predicted symptom reduction on 4 SCL-90-R scales, including somatization, obsessive-compulsive, anxiety and global severity index in patients with SAD after CBT. And high levels of agreeableness predicted symptom reduction on 2 SCL-90-R scales, including Hostility and Paranoid Ideation. High levels of openness predicted psychoticism. Conclusion: The present study suggested that high levels of three NEO-FFI dimensions (openness, agreeableness, conscientiousness) might predict comorbid psychological symptoms reduction in SAD patients after CBT. For the purpose of improving comorbid psychological symptoms with SAD patients, it might be useful to pay more attention to these dimensions of NEO-FFI at baseline.
\end{abstract}

\section{Keywords}

Social Anxiety Disorder, Cognitive-Behavioral Therapy, Comorbid Psychological Symptoms 


\section{Introduction}

Social anxiety disorder (SAD) is one of the most common psychiatric disorders with lifetime prevalence of $12 \%$ [1]. Epidemiological studies have established that psychiatric comorbidity is frequent in SAD patients [1]. SAD patients with other psychiatric disorders are associated with increased symptom severity [2].

The efficacy of cognitive-behavioral therapy (CBT) encompassing exposure therapy and cognitive restructuring has been established for SAD [3]. There is now evidence indicating that CBT for a targeted anxiety disorder yields positive benefits upon comorbid disorders [4] [5]. Predictors of less effective treatment may save patients' time by avoiding ineffective treatments, which may be sometimes associated with economic burden [6]. However, few studies have addressed predictors of outcomes in comorbid psychological symptoms after CBT for SAD.

Some studies suggest that personality mediates part of comorbidity [7] [8]. Whether personality characteristics have an impact on CBT outcome is also an important question. In CBT for SAD, however, research to identify predictive personality characteristics has been limited.

The purpose of the present study is to examine the predictive personality characteristics of comorbid psychiatric symptoms in CBT for SAD.

\section{Materials and Methods}

\subsection{Participants}

One hundred forty-four SAD patients attended the group CBT program. All of the patients met the following entry criteria: 1) principal Axis I diagnosis of SAD according to the DSM-IV criteria, as assessed by the Structured Clinical Interview for DSM-IV(SCID) [9]; 2) absence of current psychosis, bipolar disorder and substance-use disorder. The patients provided their written informed consent after receiving full explanation of the study's purpose and procedures. The study was performed in accordance with the Declaration of Helsinki and the study's protocol was approved by the Ethics Committee of our institute.

\subsection{Treatment}

The group CBT for SAD at our department was originally based on the programme developed by Andrews et al. [10]. The program consists of the following components: 1) psychoeducation; 2) behavioral experiments; 3) attention training; 4) cognitive restructuring; and 5) in vivo graded exposures. The program is run in 16 2-h weekly sessions by two therapists.

\subsection{Measures}

At pre- and post-treatment the Symptom Checklist-90 Revised (SCL-90-R) and the Liebowitz Social Anxiety Scale (LSAS) were assessed. The NEO Five Factor Index (NEO-FFI) was assessed at pre-treatment.

The SCL-90-R is a widely used and self-reported assessment tool for general psychopathology [11]. It contains 90 items, subdivided into nine subscales of somatization, obsessive-compulsive, interpersonal sensitivity, depression, anxiety, hostility, phobic anxiety, paranoid ideation, psychosis and global severity index (GSI). Each item is scored between 0 (not at all) and 5 (extremely), and the average of the relevant items was taken to be the subscale score [11].

The NEO-FFI is a 60-item self-reported questionnaire designed to measure the five major personality dimensions of neuroticism, extraversion, conscientiousness, openness and agreeableness [12]. There are 12 items per dimension and the items are answered on a 5-point scale ranging from 0 (strongly disagree) to 4 (strongly agree) [12].

The LSAS is the most frequently used clinician-administered instrument for assessment of social anxiety disorder [13]. It is a 24-item scale that provides separate scores for fear (0 - 3 indicate none, mild, moderate, and severe, respectively) and avoidance ( 0 - 3 indicate never, occasionally, often, and usually, respectively) of social interaction and performance situations [13].

\subsection{Statistical Analysis}

All the data were examined using SPSS 18.0 for Windows [14]. First, we used an independent samples t-test or 
$\chi^{2}$ test to compare the demographic and clinical data among the patients who completed the program and those who did not. Second, to examine the predictors of the indices of psychological comorbidity, we performed stepwise multiple linear regression analysis using age, sex, onset, total score of LSAS at baseline, subscales of SCL-90-R at baseline and five personality dimensions of NEO-FFI, involving neuroticism, extraversion, conscientiousness, openness and agreeableness as independent variables and subscales of SCL-90-R at endpoint as dependent variables. All the statistical tests were two-tailed, and $p \leq 0.05$ was considered statistically significant.

\section{Results}

\subsection{Patients Characteristics}

Thirty patients (26.3\%) out of the 144 who started the treatment dropped out prematurely from the CBT program and 114 patients were included in the current analysis. The reasons for dropouts were mainly the increased anxiety and the difficulties in this therapy to pursue. In baseline demographic and clinical characteristics, no statistically significant differences were seen among the subgroups (Table 1).

\subsection{Predictors of the Comorbid Psychiatric Symptoms}

In regression analysis (Table 2), high levels of Conscientiousness of NEO-FFI at baseline predicted symptom reduction on 4 SCL-90-R scales, including somatization, obsessive-compulsive, anxiety, and GSI at endpoint. And high levels of agreeableness predicted symptom reduction on 2 SCL-90-R scales, including hostility and paranoid ideation. High levels of openness predicted psychoticism. In demographic variables, sex (female) predicted symptom reduction on interpersonal sensitivity. Neuroticism, extraversion, and LSAS at baseline predicted nothing significantly.

\section{Discussion}

The present study suggests that high levels of three dimensions of NEO-FFI (openness, agreeableness, conscientiousness) at baseline may predict comorbid psychological symptoms reduction in patients with SAD after CBT. Especially high levels of conscientiousness may predict symptom reduction on somatization, obsessive-compulsive, anxiety and GSI at endpoint.

Although number of studies has examined the role of particular variables in predicting response to treatment for SAD, the results were inconsistent and inconclusive [15]. From the point of view of group therapy, our findings concerning openness and conscientiousness are consistent with those of Ogrodniczuk et al. (2003), who found openness and conscientiousness were directly associated with favorable outcome in group psychotherapy for psychiatric outpatients [16].

High openness patients are more likely benefit from group psychotherapy by being able to embrace the novel experience that psychotherapy offers [16]. High agreeableness is related favorable outcome in psychotherapy because high agreeableness patients are trusting, sympathetic, and cooperative [16]. High conscientiousness

Table 1. Baseline characteristics and mean clinical scores.

\begin{tabular}{cccc}
\hline & Completer & Dropout \\
$(\mathrm{N}=114)$ & $30)$ & $P$ value \\
Mean age (SD) & $33.4(10.5)$ & $46.7 \%$ & 0.23 \\
Sex (Male, \%) & $50 \%$ & $16.7(5.9)$ & 0.75 \\
Mean age of onset (SD) & $18.9(7.8)$ & $77.1(22.5)$ & 0.10 \\
LSAS (SD) & $75.3(25.8)$ & $29.6(8.2)$ & 0.24 \\
NEO-FFI Neuroticism (SD) & $31.6(8.4)$ & $22.3(6.5)$ & 0.60 \\
NEO-FFI Extraversion (SD) & $21.5(8.2)$ & $29.9(5.6)$ & 0.36 \\
NEO-FFI Openness (SD) & $28.8(7.1)$ & $22.1(8.0)$ & 0.06 \\
\hline
\end{tabular}

Note: LSAS, Liebowitz social anxiety scale; NEO-FFI, NEO five factor index ; SD, Standard deviation. 
Table 2. Predictors at baseline for comorbid psychological symptoms after CBT $(\mathrm{N}=114)$.

\begin{tabular}{|c|c|c|c|c|c|c|c|c|c|c|}
\hline & SOM & $\mathrm{O}-\mathrm{C}$ & I-S & DEP & ANX & HOS & РHOB & PAR & PSY & GSI \\
\hline Baseline & $0.64^{* *}$ & $0.73^{* *}$ & $0.59^{* *}$ & $0.61^{* *}$ & $0.54^{* *}$ & $0.51^{* *}$ & $0.69^{* *}$ & $0.46^{* *}$ & $0.56^{* *}$ & $0.63^{* *}$ \\
\hline Sex & a & $\mathrm{a}$ & $0.17^{*}$ & a & $\mathrm{a}$ & $\mathrm{a}$ & $\mathrm{a}$ & $\mathrm{a}$ & $\mathrm{a}$ & $\mathrm{a}$ \\
\hline Age & a & $\mathrm{a}$ & a & a & a & $\mathrm{a}$ & $\mathrm{a}$ & a & a & a \\
\hline Onset & $\mathrm{a}$ & a & a & a & a & $\mathrm{a}$ & a & $\mathrm{a}$ & a & $\mathrm{a}$ \\
\hline LSAS & a & $\mathrm{a}$ & a & a & a & a & a & a & a & a \\
\hline Neuroticism & a & $\mathrm{a}$ & a & a & $\mathrm{a}$ & a & a & a & a & a \\
\hline Extraversion & $\mathrm{a}$ & $\mathrm{a}$ & a & a & $\mathrm{a}$ & $\mathrm{a}$ & a & a & a & $\mathrm{a}$ \\
\hline Openness & a & $\mathrm{a}$ & a & $\mathrm{a}$ & $\mathrm{a}$ & $\mathrm{a}$ & a & a & $-0.17^{*}$ & $\mathrm{a}$ \\
\hline Agreeableness & a & $\mathrm{a}$ & a & a & $\mathrm{a}$ & $-0.28^{* * *}$ & $\mathrm{a}$ & $-0.25^{* *}$ & $\mathrm{a}$ & $\mathrm{a}$ \\
\hline Conscientiousness & $-0.15^{*}$ & $-0.16^{*}$ & a & $\mathrm{a}$ & $-0.16^{*}$ & $\mathrm{a}$ & $\mathrm{a}$ & a & a & $-0.15^{*}$ \\
\hline Adjusted R-square & 0.41 & 0.57 & 0.40 & 0.36 & 0.31 & 0.42 & 0.47 & 0.32 & 0.31 & 0.42 \\
\hline
\end{tabular}

Note: Table shows the standardized Beta coefficients $\left({ }^{*} P<0.05,{ }^{* *} P<0.01\right)$. a Entered into analysis but not selected in the multiple regression model through application of a stepwise method. Appendices: SOM, Somatization; O-C, Obsessive-compulsive; I-S, Interpersonal sensitivity; DEP, Depression; ANX, Anxiety; HOS, Hostility; PHOB, Phobic anxiety; PAR, Paranoid ideation; PSY, Psychosis; GSI, Global Severity Index; LSAS, Liebowitz Social Anxiety Scale.

patients are also more likely to benefit from psychotherapy because they work hard, tolerate discomfort, and delay gratification of impulses and desires [16].

For the purpose of improving comorbid symptoms with SAD patients, it might be useful to pay more attention to some dimensions of NEO-FFI, especially conscientiousness.

The present study has some limitations. First, we lacked follow-up data and could not refer to long-term effect of CBT for comorbid psychological symptoms. Second, this study did not include several predictors like expectancy regarding therapy or therapist [15]. Future studies should be conducted as follow-up study and place more focus on other predictors like patient expectancy.

\section{Conclusion}

The present study suggests that high levels of openness, agreeableness, and conscientiousness of NEO-FFI personality dimensions may predict some comorbid psychological symptoms reduction in SAD patients after CBT.

\section{Acknowledgements}

This study was supported by Grant-in-Aid from the Ministry of Health, Labour and Welfare, Japan.

\section{Conflict of Interest}

The authors do not have any conflict of interest to report regarding this study.

\section{References}

[1] Kessler, R.C., Chiu, W.T., Demler, O., Merikangas, K.R. and Walters, E.E. (2005) Prevalence, Severity, and Comorbidity of 12-Month DSM-IV Disorders in the National Comorbidity Survey Replication. Archives of General Psychiatry, 62, 617-627. http://dx.doi.org/10.1001/archpsyc.62.6.617

[2] Schneier, F.R., Johnson, J., Hornig, C.D., Liebowitz, M.R. and Weissman, M.M. (1992) Social Phobia. Comorbidity and Morbidity in an Epidemiologic Sample. Archives of General Psychiatry, 49, 282-288. http://dx.doi.org/10.1001/archpsyc.1992.01820040034004

[3] Acarturk, C., Cuijpers, P., van Straten, A. and de Graaf, R. (2009) Psychological Treatment of Social Anxiety Disorder: A Meta-analysis. Psychological Medicine, 39, 241-254. http://dx.doi.org/10.1017/S0033291708003590

[4] Craske, M.G., Farchione, T.J., Allen, L.B., Barrios, V., Stoyanova, M. and Rose, R. (2007) Cognitive Behavioral 
Therapy for Panic Disorder and Comorbidity: More of the Same or Less of More? Behaviour Research and Therapy, 45, 1095-1109. http://dx.doi.org/10.1016/j.brat.2006.09.006

[5] Tsao, J.C., Lewin, M.R. and Craske, M.G. (1998) The Effects of Cognitive-Behavior Therapy for Panic Disorder on Comorbid Conditions. Journal of Anxiety Disorders, 12, 357-371. http://dx.doi.org/10.1016/S0887-6185(98)00020-6

[6] Mululo, S.C.C., Menezes, G.B.D., Vigne, P. and Fontenelle, L.F. (2012) A Review on Predictors of Treatment Outcome in Social Anxiety Disorder. Revista Brasileira de Psiquiatria, 34, 92-100. http://dx.doi.org/10.1590/S1516-44462012000100016

[7] Battaglia, M., Przybeck, T.R., Bellodi, L. and Cloninger, C.R. (1996) Temperament Dimensions Explain the Comorbidity of Psychiatric Disorders. Comprehensive Psychiatry, 37, 292-298. http://dx.doi.org/10.1016/S0010-440X(96)90008-5

[8] Bienvenu, O.J., Brown, C., Samuels, J.F., Liang, K.Y., Costa, P.T., Eaton, W.W. and Nestadt, G. (2001) Normal Personality Traits and Comorbidity among Phobic, Panic and Major Depressive Disorders. Psychiatry Research, 102, 7385. http://dx.doi.org/10.1016/S0165-1781(01)00228-1

[9] First, M.B. (1997) Structured Clinical Interview for DSM-IV Axis I Disorders : SCID-I : Clinician Version: Administration Booklet. American Psychiatric Press, Washington DC.

[10] Andrews, G., Creamer, M., Crino, R., Hunt, C., Lampe, L. and Page, A. (2003) The Treatment of Anxiety Disorders: Clinician Guides and Patient Manuals. 2nd Edition, Cambridge University Press, Cambridge and New York.

[11] Derogatis, L.R. (1992) SCL-90-R: Administration, Scoring \& Procedures Manual-II, for the R (Revised) Version and Other Instruments of the Psychopathology Rating Scale Series. 2nd Edition, Clinical Psychometric Research, Inc., Towson.

[12] Costa, P.T. and McCrae, R.R. (1992) Revised Neo Personality Inventory (NEO PI-R) and Neo Five-Factor Inventory (NEO-FFI). Psychological Assessment Resources, Inc., Odessa.

[13] Fresco, D.M., Coles, M.E., Heimberg, R.G., Liebowitz, M.R., Hami, S., Stein, M.B. and Goetz, D. (2001) The Liebowitz Social Anxiety Scale: A Comparison of the Psychometric Properties of Self-Report and Clinician-Administered Formats. Psychological Medicine, 31, 1025-1035. http://dx.doi.org/10.1017/S0033291701004056

[14] SPSS (2009) SPSS for Windows (Version 18.0).

[15] Rodebaugh, T.L., Holaway, R.M. and Heimberg, R.G. (2004) The Treatment of Social Anxiety Disorder. Clinical Psychology Review, 24, 883-908. http://dx.doi.org/10.1016/j.cpr.2004.07.007

[16] Ogrodniczuk, J.S., Piper, W.E., Joyce, A.S., McCallum, M. and Rosie, J.S. (2003) NEO-Five Factor Personality Traits as Predictors of Response to Two Forms of Group Psychotherapy. International Journal of Group Psychotherapy, 53, 417-442. http://dx.doi.org/10.1521/ijgp.53.4.417.42832 